\title{
Controlled trial of cycled antibiotic prophylaxis to prevent initial Pseudomonas aeruginosa infection in children with cystic fibrosis
}

\author{
Gerdien A Tramper-Stranders, ${ }^{1}$ Tom F W Wolfs, ${ }^{2}$ Sanne van Haren Noman, ${ }^{1}$ \\ Wim M C van Aalderen, ${ }^{3}$ Ad F Nagelkerke, ${ }^{3}$ Marianne Nuijsink, ${ }^{4}$ Jan L L Kimpen, ${ }^{2}$ \\ Cornelis $\mathrm{K}$ van der $\mathrm{Ent}^{1}$
}

${ }^{1}$ Department of Paediatric Respiratory Medicine, Cystic Fibrosis Center, Wilhelmina Children's Hospital, University Medical Center Utrecht, The Netherlands

${ }^{2}$ Department of Pediatric Infectious Diseases, Wilhelmina Children's Hospital, University Medical Center Utrecht, The Netherlands

${ }^{3}$ Department of Pediatric Respiratory Medicine, Cystic Fibrosis Center North-West Netherlands, Emma Children's Hospital, Academic Medical Center and VU University Medical Center, Amsterdam, The Netherlands

${ }^{4}$ Department of Pediatric Respiratory Medicine, Juliana Children's Hospital, Haga Teaching Hospital, The Hague, The Netherlands

\section{Correspondence to} Gerdien A Tramper-Stranders, Cystic Fibrosis Center, KH.01.419.0, University Medical Center Utrecht, P 0 Box 85090, Utrecht 3508 AB, The Netherlands:

g.tramper@umcutrecht.nl

Received 18 December 2009 Accepted 21 May 2010 Published Online First 20 August 2010

\begin{abstract}
Background Initial pulmonary Pseudomonas aeruginosa infection in patients with cystic fibrosis (CF) is currently treated with intensive antibiotic therapy. At this stage, inflammation and tissue injury might have already occurred. Moreover, bacterial eradication is not always achieved. Prophylactic treatment against $P$ aeruginosa seemed to have a preventive effect in retrospective studies. A study was undertaken to establish prospectively the effect of cycled prophylactic treatment on prevention of initial $P$ aeruginosa infection in children with CF.

Methods This 3-year triple-blind randomised controlled trial included 65 children with CF without $P$ aeruginosa infection. Intervention existed of 3-monthly 3-week treatments with oral ciprofloxacin and inhaled colistin or both placebo controls. The primary outcome was $P$ aeruginosa infection. Secondary outcomes were serum anti-Pseudomonas antibodies, pulmonary function, exacerbations, chest $x$-ray scores, inflammation parameters, respiratory pathogens and antimicrobial resistance.
\end{abstract}

Results There was no difference in acquisition of $P$ aeruginosa infection between the control and treatment groups (annual incidence 14\% vs 11\%; HR 0.738, 95\% CI 0.299 to 1.822). Anti-Pseudomonas antibodies emerged earlier in the control group, but this difference had disappeared after 3 years. Chronic infection was observed in $19 \%$ of controls and $12 \%$ of treated patients. Decline in pulmonary function and other clinical outcomes did not differ between the two groups. In the treatment group, significantly fewer Gram-positive bacteria and Enterobacteriaceae were observed but there were more non- $P$ aeruginosa non-fermentative Gram-negative bacteria.

Conclusions Three-monthly cycled anti- $P$ aeruginosa prophylaxis does not reduce the risk of initial and chronic infection in $P$ aeruginosa-negative children with CF of all ages. Shifts in bacterial colonisation demand caution.

Trial Registration Number ISRCTN 11604593.

\section{INTRODUCTION}

Children with cystic fibrosis (CF) are at a high risk of acquiring pulmonary infections with Pseudomonas aeruginosa. A combination of host susceptibility and bacterial adaptation make $P$ aeruginosa hard to eradicate once established in the CF airways. ${ }^{1-3}$ Infection with $P$ aeruginosa is associated with irreversible pulmonary damage which is elicited by a primarily neutrophil-dominated inflammatory response and is a major predictor for mortality and morbidity. ${ }^{4-6}$

$P$ aeruginosa infection and pulmonary inflammation often precede respiratory symptoms; inflammation is more pronounced in infections with $P$ aeruginosa than with other organisms. ${ }^{7-10}$ Children are already infected at a young age; figures range from $28-85 \%$ at 3 years of age depending on isolation methods. ${ }^{49}$ A relation between $P$ aeruginosa infection and diminished lung function was observed in young children even when $P$ aeruginosa was apparently eradicated after initial infection. ${ }^{11}$

The initial detection of $P$ aeruginos $a$ in children with CF is currently managed with intensive antimicrobial therapy. Eradication of the pathogen from CF airways might be achieved when antibiotic treatment is initiated early after pulmonary acquisition. ${ }^{912-19}$ The optimal treatment regimen has not yet been established; there is a lack of randomised controlled trials comparing several regimens of antimicrobial therapy. ${ }^{19} 20$ Despite early antibiotic therapy, eradication fails in $10-40 \%$ of cases and these patients are predisposed to chronic infection with $P$ aeruginosa. In addition, when eradication is successful, reinfection often occurs within 1 year, putting the patient at risk for further deterioration of pulmonary function. Limited evidence from two retrospective studies indicated that continuous prophylactic antibiotic therapy against $P$ aeruginosa reduced the number of patients with initial and chronic Paeruginosa infections. ${ }^{21} 22$

The fact that $P$ aeruginosa infection in early life is associated with irreversible pulmonary damage and that early eradication therapy might fail prompted us to prospectively evaluate the effectiveness of prophylactic antibiotic therapy in preventing initial $P$ aeruginosa infection and subsequent decline in pulmonary function. We hypothesised that cycled prophylactic anti-Pseudomonas treatment of $P$ aeruginosa-negative CF patients would either prevent or delay the first acquisition of $P$ aeruginosa or eradicate the organism at a very early phase before the onset of chronic pulmonary infection, the accompanying pulmonary inflammatory response and bacterial adaptation.

\section{METHODS \\ Patients and settings}

Children aged $0-18$ years were recruited from four tertiary CF centres in The Netherlands. Eligibility criteria were CF confirmed by a positive sweat 
chloride test and/or genotyping and $P$ aeruginosa negativity determined as the absence of $P$ aeruginosa-positive cultures and/ or serology and the absence of regular treatment with antiPseudomonas antibiotics in the previous 2 years.

\section{Study design and randomisation}

We conducted an investigator-initiated parallel group randomised placebo-controlled trial with triple blinding (investigators, physicians and participants). Participants were randomly assigned by a computerised program in blocks of four, stratified according to centre and age ( $0-5$ years, 6-11 years, 12-18 years). Randomisation and packaging of study interventions was performed by the independent pharmacy department of the University Medical Center Utrecht. The randomisation code was only disclosed after completion of all follow-up data and entry of data in a study database.

\section{Study intervention}

The study duration was 3 years. The intervention consisted of inhalation of colistimethate sodium (colistin) $80 \mathrm{mg}$ twice daily combined with oral ciprofloxacin $10 \mathrm{mg} / \mathrm{kg}$ twice daily or both placebo controls for continuous periods of 3 weeks every 3 months. Within a 3 -year study period there were 12 study intervention cycles. This eradication regimen was chosen because it is used extensively as eradication therapy in children in CF centres across Europe. Colistimethate sodium powder $80 \mathrm{mg}$ (1 million units) per dose and an identical placebo containing $80 \mathrm{mg}$ mannitol powder, both with $3 \mathrm{ml}$ normal saline as solvent, were provided by Grünenthal, Aachen, Germany. Colistimethate sodium or placebo was inhaled by a perforated vibrating membrane nebuliser (eFlow Rapid, PARI, Starnberg, Germany) and, if necessary, preceded by salbutamol inhalation. ${ }^{23}$ Ciprofloxacin granules and corresponding identical placebo (coated sugar sphere granules) with sweet-flavoured suspension were provided by Bayer Schering Pharma, Berlin, Germany.

\section{Follow-up during the study}

Follow-up visits were performed 3-monthly 2-5 weeks after the study intervention. At each visit a respiratory specimen (oropharyngeal cough swab or sputum) was collected for microbial culture. The number of pulmonary exacerbations, use of antimicrobial agents, height, weight and adverse events were noted. Additionally, pharmacists were asked to provide an overview of antimicrobial agent use during the study period to prevent recall bias. Spirometric tests for children aged $\geq 4$ years were performed 6-monthly. Pulmonary function was assessed as forced expiratory volume in $1 \mathrm{~s}$ percentage predicted for height and sex $\left(\mathrm{FEV}_{1} \%\right)$ and forced vital capacity percentage predicted for height and sex (FVC\%) after inhalation of $800 \mu \mathrm{g}$ salbutamol using reference data of Zapletal and Samenek. ${ }^{24}$ Serum samples were collected every 6 months for measurement of serum antiPseudomonas antibodies. Chest radiography, complete blood count, C-reactive protein and serum immunoglobulin measurements were performed annually. Other standard CF care was maintained throughout the trial.

\section{Microbiology}

Respiratory specimens were cultured onto appropriate media and Pseudomonas species were identified by a positive oxidase reaction. Further identification of $P$ aeruginosa was performed by measuring C-390 resistance. ${ }^{25}$ Susceptibility testing was performed by disk diffusion using Neo-Sensitabs (Rosco, Taastrup, Denmark) and was interpreted according to Clinical and Laboratory Standards Institute breakpoints.
Serological testing was performed by ELISA with three common $P$ aeruginosa antigens: elastase, exotoxin A and alkaline protease (Mediagnost, Reutlingen, Germany) as described previously. ${ }^{26}$ A titre of $>1: 1250$ for one or more of the antigens was considered positive, as described in the ELISA manual.

\section{Outcomes}

The primary efficacy outcome was $P$ aeruginosa infection, as determined by two positive cultures taken $>1$ week apart (to increase the diagnostic values for pulmonary $P$ aeruginosa infection in case of oropharyngeal cough swabs) or, at the discretion of the treating physician, one positive culture and a severe pulmonary exacerbation that required rapid antimicrobial treatment. When the primary end point was reached, the participants stopped further study intervention and received standard anti-Pseudomonas treatment. All participants who reached the primary end point or who withdrew from study interventions were included in the follow-up. Serological test results were not included in the primary efficacy end point.

Secondary efficacy outcomes included anti-Pseudomonas antibodies, pulmonary function, body mass index, number of pulmonary exacerbations (defined by increased cough and/or sputum production and/or decreased weight and/or changes in chest auscultation requiring antimicrobial therapy), days of antibiotic use (all antibiotic treatments; anti-Pseudomonas antibiotics were only prescribed in cases reaching the primary efficacy outcome), adverse events, antimicrobial resistance, bacterial colonisation, chest x-ray (adjusted Crispin-Norman ${ }^{27}$ ) scores and inflammation parameters.

\section{Statistical analysis and power calculation}

It was estimated that 32 children were needed in each group for the study to have $80 \%$ power to detect a $75 \%$ reduction in $P$ aeruginosa infections in the treatment group at a two-sided $\alpha$ level of 0.05 , assuming a $P$ aeruginosa acquisition rate of $15 \%$ per year in the control group.

The trial was analysed by the principal investigator according to the intention to treat principle. In participants reaching the primary efficacy end point, data on cultured pathogens and antimicrobial agent use were analysed from the start of the study until the first full study year after the end point was reached. Survival analysis was performed using Kaplan-Meier curves and Cox proportional hazard model regression with age and baseline $\mathrm{FEV}_{1} \%$ as stratifying variables. In order to test the assumption of proportional hazards for the treatment group in the Cox model, a time-dependent variable for the group was created and tested using the likelihood ratio test. Nominal data were analysed using the $\chi^{2}$ or Fisher exact test and continuous data were analysed with the test or Mann-Whitney $U$ test. Data are shown as percentages or mean \pm SEM.

Longitudinal data with repeated measurements (pulmonary function and antibody titres) were analysed with a mixed effects model with random participant-specific slope and intercept. Baseline $\mathrm{FEV}_{1} \%$ and age were included as covariates in the pulmonary function model. An interaction term between allocation and time was examined in order to determine whether decline in pulmonary function or increase in antibody titres differed between allocation groups.

Statistical analysis was performed with SPSS Version 15.0.

\section{RESULTS}

\section{Study group}

Sixty-five patients were randomised and entered the study. Figure 1 shows the flow diagram of inclusion. Baseline 


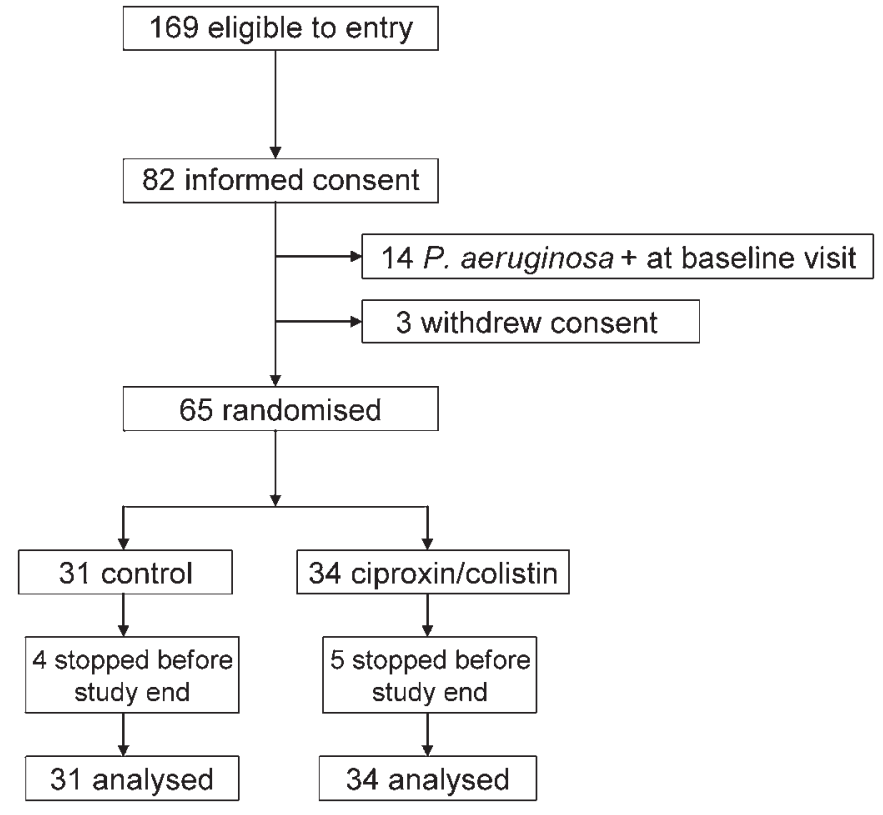

Figure 1 Flow diagram showing the recruitment and selection of participants.

characteristics for the control and treatment groups were similar for the total group as well as for different age subgroups except for $\mathrm{FEV}_{1} \%(\mathrm{p}=0.01$, table 1$)$.

\section{Pseudomonas aeruginosa infection}

Nineteen of 65 patients (29\%) reached the primary end point during the study period, 10/31 (32\%) in the control group and $9 / 34(26 \%)$ in the treatment group. The incidence rates were 0.14 for the control group and 0.11 for the treatment group. The hazard ratio (HR) for the treatment group to acquire $P$ aeruginosa infection was 0.738 (95\% CI 0.299 to 1.822 ) $(\mathrm{p}=0.510)$ stratified for age and baseline $\mathrm{FEV}_{1} \%$. Mean duration to $P$ aeruginosa infection was $0.86 \pm 0.21$ years for the control group and $1.29 \pm 0.19$ years for the treatment group $(p=0.158)$. Figure $2 \mathrm{~A}$ shows the survival curve. The HRs were not different between the groups over time (likelihood ratio test 2.682 with 1 degree of freedom, $p=0.101$ ). The median age at acquiring first $P$ aeruginosa infection was 6.8 years (range 2.45-18.42). Patients with previous $P$ aeruginosa isolation $>2$ years before study entry did not reach the primary end point more often $(\mathrm{p}=0.912)$.

Table 1 Baseline characteristics of participating children with CF without Pseudomonas aeruginosa infection

\begin{tabular}{lll}
\hline Allocation & $\begin{array}{l}\text { Control } \\
(\mathbf{n = 3 1 )}\end{array}$ & $\begin{array}{l}\text { Treatment } \\
(\mathbf{n}=\mathbf{3 4})\end{array}$ \\
\hline Mean \pm SE age (years) & $\begin{array}{l}7.79 \pm 0.68 \\
16(52 \%)\end{array}$ & $\begin{array}{l}6.69 \pm 0.67 \\
14(41.2 \%)\end{array}$ \\
Female sex & & \\
CF genotype & 19 & 22 \\
$\quad$ dF508/dF508 & 11 & 10 \\
$\quad$ dF508/other & 1 & 2 \\
$\quad$ Other/other & $38.7 \%$ & $14.7 \%$ \\
Sputum producer & $15(48 \%)$ & $13(38 \%)$ \\
Staphylococcus aureus colonisation & $21(68 \%)$ & $18(53 \%)$ \\
Antistaphylococcal prophylaxis & $6(19.4 \%)$ & $7(20.6 \%)$ \\
Ever $P$ aeruginosa in culture $>2$ years before study entry & $94 \pm 3.0$ & $107 \pm 3.7$ \\
Mean \pm SE FEV $\%$ & & \\
Mean \pm SE BMl z-Score & $-0.16 \pm 0.17$ & $0.25 \pm 0.14$ \\
\hline
\end{tabular}

BMI, body mass index; $\mathrm{FEV}_{1}$, forced expiratory volume in $1 \mathrm{~s}$.
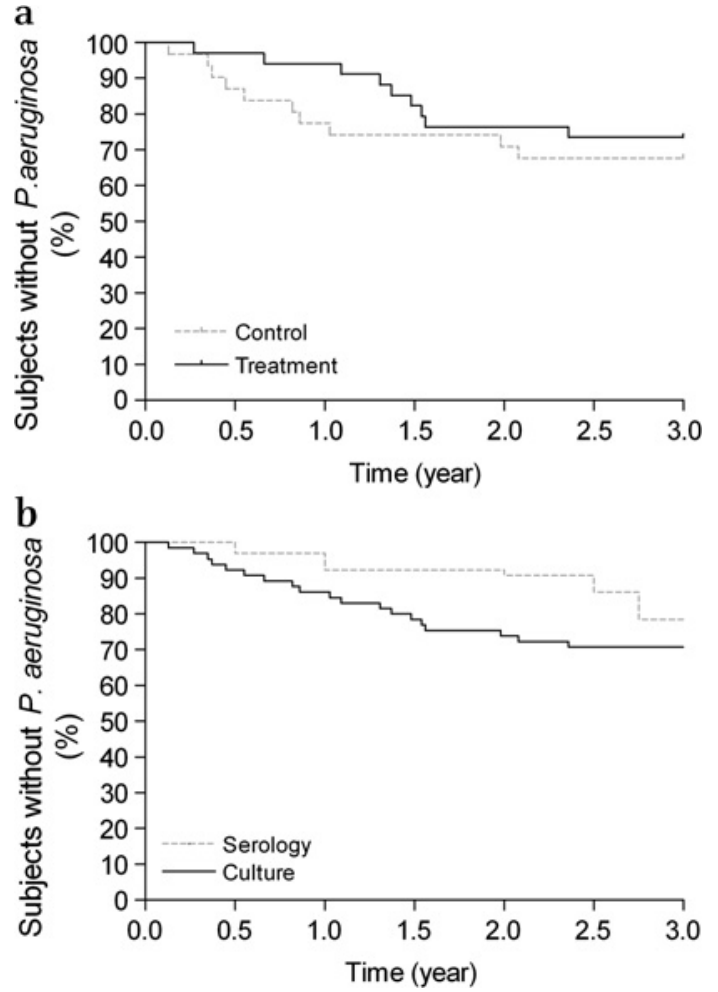

Figure 2 Kaplan-Meier survival curves of $(A)$ the primary efficacy end point and $(B)$ for the entire study group with respect to timing of culture positivity and serology positivity.

Of the 19 participants who reached the primary efficacy end point, four had one $P$ aeruginosa-positive swab culture accompanied by an exacerbation. Three of the four patients with an exacerbation had a second positive culture subsequently. In four of the 19 patients $P$ aeruginosa was detected in the sputum ( 3 in the control group, 1 in the treatment group). Three other participants had $P$ aeruginosa in a swab culture on one occasion without an exacerbation, follow-up cultures remained negative and therefore the primary end point was not met.

Eradication of $P$ aeruginosa in patients who had reached the primary end point and ceased the study interventions to receive usual anti-Pseudomonas treatment was established in $60 \%$ of patients in the control group and $67 \%$ of patients in the treatment group after one or more eradication cycles $(p=0.764)$.

\section{Pseudomonas aeruginosa serology}

The proportion of patients having a positive antibody titre was higher in the control group during the first year $(p=0.021)$; in the second and third years the differences between the two groups disappeared. As calculated by a mixed model, the sum of three serum anti-Pseudomonas antibodies was not different $(p=0.08)$. In general, positive antibody titres followed positive cultures and were strongly associated with a positive culture in the past (figure 2B). Fourteen children had positive antibody titres at least once during the study period: exotoxin A antibody titres were raised in 10 patients, alkaline protease in five patients and elastase in three patients. Ten of the 14 children with positive antibody titres were culture-positive before they were serology-positive. Two patients showed intermittent positive antibody titres without any positive cultures during the study period and two other patients showed positive antibody titres without positive cultures to date (two in the control group and two in the treatment group). 
Table 2 Outcome of clinical parameters

\begin{tabular}{llll}
\hline & Control & Treatment & p Value \\
\hline FEV $_{1} \%$ decline during study period & $-8.3 \%$ & $-10.4 \%$ & 0.940 \\
BMl z-score change & $-0.09 \pm 0.15$ & $-0.003 \pm 0.08$ & 0.603 \\
Chest x-ray score change & $+2.10 \pm 0.51$ & $+2.00 \pm 0.50$ & 0.885 \\
Inflammatory parameters & & & \\
$\quad$ Immunoglobulin G & $9.84 \pm 3.54$ & $8.66 \pm 3.82$ & 0.105 \\
$\quad$ Leucocytes ( $\times 10^{9} /$ l) & $9.17 \pm 2.88$ & $8.67 \pm 2.68$ & 0.499 \\
$\quad$ Proportion of patients with CRP & $16.1 \%$ & $14.7 \%$ & 0.874 \\
$\quad>10$ mg/l during study & & & \\
& & & \\
Adverse events & $45.2 \%$ & $50.0 \%$ & 0.696 \\
$\quad$ Cough and/or increased sputum production & $38.7 \%$ & $23.5 \%$ & 0.185 \\
$\quad$ Gastrointestinal & $9.7 \%$ & $20.6 \%$ & 0.192 \\
Other (headache, erythema, fatigue, & $0.0 \%$ & $14.7 \%$ & 0.054 \\
incontinence) & & & \\
\hline
\end{tabular}

Chronic infection, defined as $>50 \%$ of evaluable months $P$ aeruginosa in cultures for at least 1 year and raised antibody titres in the last study year, was observed in $19 \%$ of patients in the control group and $12 \%$ of patients in the treatment group $(\mathrm{p}=0.500)$.

\section{Secondary clinical outcomes}

If corrected for baseline $\mathrm{FEV}_{1} \%$ and age, the average $\mathrm{FEV}_{1} \%$ was not higher in the treatment group during the study $(\mathrm{p}=0.206)$. The rate of decline in pulmonary function $\left(\mathrm{FEV}_{1} \%\right.$ ) over the 3 -year study period in the treatment group was not significantly different from the control group $(p=0.94)$, being $-8.3 \%$ for the control group and $-10.4 \%$ for the treatment group. Older children did not have a faster decline in $\mathrm{FEV}_{1} \%$ than younger children. In the first study year $67.7 \%$ of the control group and $58.8 \%$ of the treatment group required one or more antibiotic treatments because of a pulmonary exacerbation or reaching the primary end point. The median time to first exacerbation requiring antibiotic therapy was 0.75 years for the control group and 1.00 year for the treatment group $(p=0.223)$. The number of intravenous antibiotic treatments during the 3-year study period did not differ between groups (17\% in the control group vs $14 \%$ in the treatment group). The mean number of days on antimicrobial therapy because of respiratory exacerbations (excluding maintenance antibiotics) for the total study period was $66 \pm 9.52$ for the control group and $67 \pm 9.92$ for the treatment group. The results for the other secondary outcomes are shown in table 2.

\section{Antimicrobial resistance and emerging pathogens}

No ciprofloxacin or colistin resistance was observed in any initial infecting $P$ aeruginosa isolates. One isolate from both groups was, respectively, aminoglycoside-resistant and $\beta$-lactam-resistant.

Enterobacteriaceae and Gram-positive bacteria were more frequently detected in cultures from the control group (table 3).
The percentage of cultures containing non-fermenting Gramnegative bacteria excluding $P$ aeruginosa was significantly higher in the treatment group $(p=0.0094)$. The presence of Candida spp. and fungi was not increased in the treatment group.

\section{DISCUSSION}

This first prospective study of the prophylaxis of initial $P$ aeruginosa infection by cycled antibiotic treatment failed to show a preventive effect in the entire group of children aged 0-18 years. Anti-Pseudomonas antibodies emerged later in the treated group, but this difference had disappeared after 3 years.

Two previous observational retrospective studies ${ }^{21} 22$ suggested a preventive effect of the use of anti-Pseudomonas prophylaxis. However, these studies were not controlled and the group of children treated twice daily with inhaled anti-Pseudomonas antibiotics was different from the group of untreated children. The approach in the current controlled trial was cycled treatment rather than daily treatment to limit the patient burden and the risk of antimicrobial resistance. It appeared that 3-monthly cycled therapy was not sufficiently intense to prevent initial $P$ aeruginosa infection. Continuous prophylaxis seems to have a better effect on the prevention of initial $P$ aeruginosa infection. In the first part of the study the survival lines of the two study groups diverged, indicating an emerging difference between the groups and postponement of infection in the treatment group. However, after 1.5 years there was a decline in the survival curve of the treatment group and the survival curves became more or less parallel. This difference in HRs over time was not statistically significant. We could not find a clear reason for this steep decline, which occurred in patients in all age categories. Reported compliance was not different, but study intervention packages were not returned so compliance was not measured. We wanted to reflect a 'real life' situation and it is expected that, in this situation, compliance is not always optimal.

Viral infections, especially the respiratory syncytial virus, predispose to pulmonary $P$ aeruginosa infection. ${ }^{28}$ In this study 13 of the 19 children (68\%) acquired $P$ aeruginosa infection between the months of October to March, suggesting a role for viruses. Continuous prophylaxis during the winter season or a cycle when experiencing a viral infection might be better prevention for $P$ aeruginosa infections than cycled treatments over all the year. The number of exacerbations and the use of antimicrobial agents were not significantly different between the two groups. A possible reason for the lack of differences is that, in young children, viral infections often induce respiratory exacerbations but antimicrobial treatment is not withheld because of the risk of bacterial infection. It was recently shown that children with CF often have more symptoms from viral respiratory infections than healthy controls. ${ }^{29}$

About 29\% of the patients in our study group reached the efficacy end point within 3 years (incidence rate $12 \%$ ). The

Table 3 Frequency of bacterial pathogens in respiratory cultures

\begin{tabular}{|c|c|c|c|c|c|c|}
\hline & Control (N) & Treatment (N) & Control $(\mathrm{N}=308)$ & Treatment $(\mathrm{N}=368)^{*}$ & Risk difference (95\% CI) & $\overline{p \text { Value }}$ \\
\hline Gram-positive bacteria & 189 & 188 & $61.36 \%$ & $51.09 \%$ & 10.3 (2.8 to 17.8$)$ & 0.007 \\
\hline Gram-negative bacteria & 97 & 113 & $36.36 \%$ & $36.14 \%$ & $0.8(-6.2$ to 7.8$)$ & 0.83 \\
\hline Non-fermenting bacteria & 24 & 52 & $7.79 \%$ & $14.13 \%$ & $6.4(1.6$ to 11.1$)$ & 0.009 \\
\hline Other Gram-negative bacteria & 45 & 43 & $14.61 \%$ & $11.68 \%$ & $2.9(-2.2$ to 8.0$)$ & 0.26 \\
\hline Yeast and fungi & 152 & 190 & $49.35 \%$ & $51.63 \%$ & $2.3(-5.3$ to 9.8$)$ & 0.56 \\
\hline
\end{tabular}

*Differences explained by the fact that, in participants reaching the primary efficacy end point, data were analysed from the start of the study until the first full study year after having reached the end point. 
power analysis was executed assuming a 15\% annual acquisition rate and $75 \%$ reduction in $P$ aeruginosa infections in the treated group. With a larger study group it would have been possible to find smaller treatment differences. However, in the $0-5$ years age group the incidence rate was $17 \%$ per year with no difference between the control and treatment groups (HR 1.028, $\mathrm{p}=0.967$ ). In the power calculation we chose a $75 \%$ reduction because smaller treatment differences would probably not justify the treatment burden and risks compared with regular culturing and intensive treatment of initial infection.

It is debatable whether the primary end point should be chronic infection. However, if prevention of initial infection occurs, inflammation and possible progression to chronic infection are delayed. With regard to the definitions of chronic infection, patients in the control group had raised antibody titres indicative of chronic infection earlier, but the difference disappeared after 1 year. The number of patients with chronic infection at the end of the trial did not differ between the groups. Serological measurements did not contribute much to the detection of the initial infection. The timing of emergence of antibodies depends also on the antigens tested. Whole cell ELISA or type 3 system secretion products seem to be more sensitive than the antigens used in this study. ${ }^{30} 31$ However, positive serological results do not precede a positive culture in every patient.

Mannitol, an osmolytic agent, was used as control inhalation medication. At the time of the study design, mannitol was not known as an airway clearance promoter for patients with CF. Nowadays, clinical trials have been initiated to prove the effect of mannitol inhalation on pulmonary function and number of exacerbations. ${ }^{32}$ It is not sure whether mannitol has a preventive effect on acquisition of pathogens in the airways because of better airway clearance. Pulmonary function decline in a third group of patients (eligible patients who decided not to participate in the study) was estimated and was not different from the control group.

Intensive anti-Pseudomonas antimicrobial treatment might lead to selection of highly resistant bacteria such as Stenotrophomonas maltophilia and Achromobacter xylosoxidans. The clinical importance of this finding is still unknown but should raise awareness among physicians. ${ }^{33}$ Gram-negative nonfermenting bacteria as a group (including $S$ maltophilia, Acinetobacter spp. and Burkholderia cepacia complex) were more frequently detected in cultures from the treatment group. Candida species were not detected more frequently in the treatment group, probably reflecting the overall high use of antimicrobial agents among patients with CF.

\section{CONCLUSION}

Three-monthly cycled anti-Pseudomonas prophylaxis in Paeruginosanegative children with CF does not prevent initial pulmonary $P$ aeruginosa infection. Frequent culturing and early intensive antimicrobial therapy when $P$ aeruginosa is detected is probably the best option, especially in view of the treatment burden and emerging pathogens. Efforts should be made to identify children or situations with an additional risk for initial and chronic $P$ aeruginosa infection.

Acknowledgements The authors thank S Michel for assistance with data gathering, J Kouwenberg for patient inclusion, $\mathrm{J}$ van der Bruggen for assistance with bacterial typing and $\mathrm{R} \mathrm{K}$ Stellato for statistical advice.

Funding This study was funded by the Dutch Cystic Fibrosis Foundation. Study medication was provided by Bayer and Grunenthal who did not participate in the design, analysis or writing of the article.

\section{Competing interests None}

Ethics approval This study was conducted with the approval of the medical ethica committees of the University Medical Centre Utrecht, Academical Medical Centre Amsterdam, VU Medical Centre Amsterdam, Haga Teaching Hospital, The Hague. Parents and children aged $>12$ years provided written informed consent before study participation.

Provenance and peer review Not commissioned; externally peer reviewed.

\section{REFERENCES}

1. Ratjen F, Doring G. Cystic fibrosis. Lancet 2003;361:681-9.

2. Sadikot RT, Blackwell TS, Christman JW, et al. Pathogen-host interactions in Pseudomonas aeruginosa pneumonia. Am J Respir Crit Care Med 2005;171:1209-23

3. Nguyen D, Singh PK. Evolving stealth: genetic adaptation of Pseudomonas aeruginosa during cystic fibrosis infections. Proc Natl Acad Sci USA 2006;103:8305-6.

4. Li Z, Kosorok MR, Farrell PM, et al. Longitudinal development of mucoid Pseudomonas aeruginosa infection and lung disease progression in children with cystic fibrosis. JAMA 2005:293:581-8.

5. Emerson J, Rosenfeld M, McNamara S, et al. Pseudomonas aeruginosa and other predictors of mortality and morbidity in young children with cystic fibrosis. Pediatr Pulmonol 2002:34:91-100.

6. Kosorok MR, Zeng L, West SE, et al. Acceleration of lung disease in children with cystic fibrosis after Pseudomonas aeruginosa acquisition. Pediatr Pulmonol 2001;32:277-87.

7. Burns JL, Gibson RL, McNamara S, et al. Longitudinal assessment of Pseudomonas aeruginosa in young children with cystic fibrosis. J Infect Dis 2001;183:444-52

8. Dakin CJ, Numa $\mathrm{AH}$, Wang $\mathrm{H}$, et al. Inflammation, infection, and pulmonary function in infants and young children with cystic fibrosis. Am J Respir Crit Care Med 2002;165:904-10.

9. Douglas TA, Brennan $S$, Gard $S$, et al. Acquisition and eradication of $P$. aeruginosa in young children with cystic fibrosis. Eur Respir J 2009;33:305-11.

10. Sagel SD, Gibson RL, Emerson J, et al. Impact of Pseudomonas and Staphylococcus infection on inflammation and clinical status in young children with cystic fibrosis. $J$ Pediatr 2009;154:183-8.

11. Kozlowska WJ, Bush A, Wade A, et al. Lung function from infancy to the preschoo years after clinical diagnosis of cystic fibrosis. Am J Respir Crit Care Med 2008;178:42-9.

12. Valerius NH, Koch C, Hoiby N. Prevention of chronic Pseudomonas aeruginosa colonisation in cystic fibrosis by early treatment. Lancet 1991;338:725-6.

13. Wiesemann HG, Steinkamp G, Ratjen F, et al. Placebo-controlled, doubleblind, randomized study of aerosolized tobramycin for early treatment of Pseudomonas aeruginosa colonisation in cystic fibrosis. Pediatr Pulmonol 1998;25:88-92.

14. Ratjen F, Doring G, Nikolaizik WH. Effect of inhaled tobramycin on early Pseudomonas aeruginosa colonisation in patients with cystic fibrosis. Lancet 2001;358:983-4

15. Gibson RL, Emerson J, McNamara S, et al. Significant microbiological effect of inhaled tobramycin in young children with cystic fibrosis. Am J Respir Crit Care Med 2003:167:841-9

16. Gibson RL, Emerson J, Mayer-Hamblett N, et al. Duration of treatment effect afte tobramycin solution for inhalation in young children with cystic fibrosis. Pediatr Pulmonol 2007:42:610-23.

17. Taccetti G, Campana S, Festini F, et al. Early eradication therapy agains Pseudomonas aeruginosa in cystic fibrosis patients. Eur Respir J 2005;26:458-61.

18. Littlewood JM, Miller MG, Ghoneim AT, et al. Nebulised colomycin for early pseudomonas colonisation in cystic fibrosis. Lancet 1985;1:865.

19. Ratjen F. Treatment of early Pseudomonas aeruginosa infection in patients with cystic fibrosis. Curr Opin Pulm Med 2006;12:428-32.

20. Jones AM. Eradication therapy for early Pseudomonas aeruginosa infection in CF: many questions still unanswered. Eur Respir J 2005;26:373-5.

21. Heinzl B, Eber E, Oberwaldner B, et al. Effects of inhaled gentamicin prophylaxis on acquisition of Pseudomonas aeruginosa in children with cystic fibrosis: a pilot study. Pediatr Pulmonol 2002:33:32-7.

22. Lebecque $\mathbf{P}$, Leal T, Zylberberg K, et al. Towards zero prevalence of chronic Pseudomonas aeruginosa infection in children with cystic fibrosis. J Cyst Fibros 2006:5:237-44.

23. Bitterle E. Comparison of aerosol delivery efficiency nebulising colistin by electronic and jet nebulisers. 2009. http://ddl-conference.org.uk/files/posters/44.Tservistas.pdf laccessed 29 Jul 2010)

24. Zapletal A, Samenek TP. Lung function in children and adolescents: methods and reference values. Basel, Munchen: Karger, 1987.

25. Campbell ME, Farmer SW, Speert DP. New selective medium for Pseudomonas aeruginosa with phenanthroline and 9-chloro-9-[4-(diethylamino) phenyl]-9,10-dihydro-10- phenylacridine hydrochloride (C-390). J Clin Microbiol 1988;26:1910-12.

26. Tramper-Stranders GA, van der Ent CK, Slieker MG, et al. Diagnostic value of serological tests against Pseudomonas aeruginosa in a large cystic fibrosis population. Thorax 2006;61:689-93.

27. van der Put JM, Meradji M, Danoesastro D, et al. Chest radiographs in cystic fibrosis. A follow-up study with application of a quantitative system. Pediatr Radiol 1982;12:57-61. 
28. van Ewijk BE, Wolfs TF, Fleer $A$, et al. High Pseudomonas aeruginosa acquisition rate in CF. Thorax 2006;61:641-2

29. van Ewijk BE, van der Zalm MM, Wolfs TF, et al. Prevalence and impact of respiratory viral infections in young children with cystic fibrosis: prospective cohort study. Pediatrics 2008;122:1171-6.

30. Ratjen F, Walter $\mathrm{H}$, Haug $\mathrm{M}$, et al. Diagnostic value of serum antibodies in early Pseudomonas aeruginosa infection in cystic fibrosis patients. Pediatr Pulmonol 2007; 42:249-55.
31. West SE, Zeng L, Lee BL, et al. Respiratory infections with Pseudomonas aeruginosa in children with cystic fibrosis: early detection by serology and assessment of risk factors. JAMA 2002;287:2958-67.

32. Jaques A, Daviskas $E$, Turton JA, et al. Inhaled mannitol improves lung function in cystic fibrosis. Chest 2008;133:1388-96.

33. Waters V, Ratjen F. Multidrug-resistant organisms in cystic fibrosis: management and infection-control issues. Expert Rev Anti Infect The 2006:4:807-19.

\section{Pulmonary puzzle}

\section{ANSWER}

From the questions on page 890

Analysis of bronchoalveolar lavage from the right $\mathrm{B}^{5}$ a segment revealed a total cell count increase of $7.8 \times 10^{5} / \mathrm{ml}$ with $50 \%$ eosinophils. No bacteria or tubercle bacilli were detected in the bronchoalveolar lavage fluid. Eosinophilic infiltration with degranulation and fibrin was seen in the transbronchial lung biopsy specimen (figure 1A,B). These histopathological findings are consistent with eosinophilic pneumonia (EP).

Oesophagogastroduodenoscopy revealed an Anisakis simplex on the gastric body mucosa. After removal of the parasite with endoscopic biopsy forceps (figure 2), the patient's severe epigastralgia, dyspnoea, cough, fever and respiratory failure improved almost immediately without steroid treatment. Radiographic abnormalities improved remarkably at 3 days after removal of the Anisakis. Because improvement of the patient's EP occurred only after removal of the Anisakis, our ultimate diagnosis was EP due to gastric anisakiasis.

Although various parasitic diseases can cause EP, ${ }^{1}$ there have been no reports implicating Anisakis. Anisakiasis can be responsible for four clinical forms of illness in humans: gastric, intestinal and ectopic anisakidosis, and allergic forms. ${ }^{2}$ Three cases have been reported in which the Anisakis was located in the intrathoracic area rather thanthe gastrointestinal tract. ${ }^{3-5}$ Allergic patients suffering from anaphylaxis can exhibit bronchospasm, urticaria, angioneurotic oedema and digestive symptoms. $^{2}$
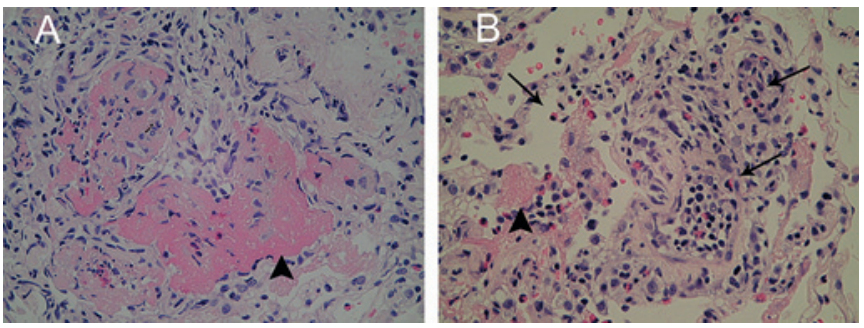

Figure 1 Histopathological findings of transbronchial lung biopsy. Eosinophilic infiltration (arrows) and fibrin depositions (arrowheads) are seen. (A) $\times 20$ magnification; (B) $\times 40$ magnification.

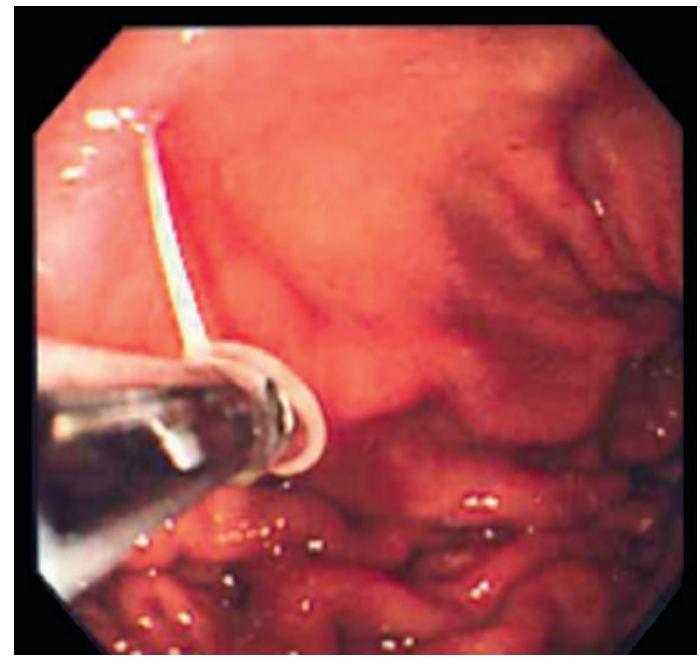

Figure 2 Oesophagogastroduodenoscopy revealed an Anisakis at the gastric corpus. The Anisakis was removed with endoscopic biopsy forceps.

Our patient frequently ate raw seafood such as squid and saba (mackerel) before admission and was positive for the $A$ simplex antibody by radioallergosorbent test. Therefore, we thought this to be a case of fulminant-type gastric anisakiasis with sensitisation and Arthus-type hypersensitivity reaction. Such allergic reactions might possibly cause EP.

Thorax 2010;65:920. doi:10.1136/thx.2010.136655

\section{REFERENCES}

1. Chitkara RK, Krishna G. Parasitic pulmonary eosinophilia. Semin Respir Crit Care Med 2006;27:171-84.

2. Audicana MT, Kennedy MW. Anisakis simplex: from obscure infectious worm to inducer of immune hypersensitivity. Clin Microbiol Rev 2008:21:360-79.

3. Matsuoka H, Nakama T, Kisanuki $\mathrm{H}$, et al. A case report of serologically diagnosed pulmonary anisakiasis with pleural effusion and multiple lesions. Am J Trop Med Hyg 1994:51:819-22.

4. Kobayashi A, Tsuji M, Wilbur DL. Probable pulmonary anisakiasis accompanying pleural effusion. Am J Trop Med Hvg 1985;34:310-13.

5. Saito W, Kawakami K, Kuroki R, et al. Pulmonary anisakiasis presenting as eosinophilic pleural effusion. Respirology 2005;10:261-2 